\title{
Tailoring Nonlinearity and Dispersion of Photonic Crystal Fibers Using Hybrid Cladding
}

\author{
Liu Zhao-lun, ${ }^{*}$ Hou Lan-tian, and Wang Wei \\ Institute of Infrared Optical Fibers and Sensors, Yanshan University, Qinhuangdao, 066004, China
}

(Received on 5 October, 2008)

\begin{abstract}
We present a hybrid cladding photonic crystal fiber for shaping high nonlinear and flattened dispersion in a wide range of wavelengths. The new structure adopts hybrid cladding with different pitches, air-holes diameters and air-holes arrayed fashions. The full-vector finite element method with perfectly matched layer is used to investigate the characteristics of the hybrid cladding photonic crystal fiber such as nonlinearity and dispersion properties. The influence of the cladding structure parameters on the nonlinear coefficient and geometric dispersion is analyzed. High nonlinear coefficient and the dispersion properties of fibers are tailored by adjusting the cladding structure parameters. A novel hybrid cladding photonic crystal fiber with high nonlinear coefficient and dispersion flattened which is suited for supercontinuum generation is designed.
\end{abstract}

Keywords: Photonic crystal fiber, nonlinearity, dispersion, hybrid cladding, finite element method

\section{INTRODUCTION}

It is well known that photonic crystal fibers (PCFs) or microstructured fibers or holey fibers which are optical fibers with a complex microstructure in the transverse plane, of various types, can offer advantage in the design of fiber [1,2]. PCFs have emerged as an attractive researching object within the area of fiber functional devices. Such holey claddings in PCFs help controlling nonlinear coefficient and tuning dispersion slope in a way that was not possible in conventional fibers [3-5]. In such PCFs, control of chromatic dispersion keeping high nonlinear coefficient is crucial for practical applications to nonlinear optics.

High nonlinear PCFs are perfect nonlinear optical medium, and the study of the nonlinearity of PCFs has been an important branch in the field of nonlinear optics [6-8]. Using high nonlinear PCFs, nonlinear effects including supercontinuum generation and frequent conversion can be realized easily [912]. High nonlinear PCFs can be efficiently used to generate supercontinuum pumped by ultra fast laser pulses and longer laser pulses. However, supercontinuum generation in PCFs is restricted by dispersion properties. Therefore, not only extremely high nonlinear coefficient is necessary, but also flattened dispersion and low loss are desired [13-15]. In this paper, we propose a hybrid cladding PCFs which has high nonlinearity and flattened dispersion. The research is predominantly aimed at the design of PCFs with small core and proper cladding structure parameters to obtain high nonlinearity and some dispersion properties. One interesting finding from our simulation is that we can obtain high nonlinear coefficient of PCFs by using hybrid cladding and adjusting the cladding structure parameters.

\section{HYBRID CLADDING DESIGN}

In conventional PCFs, the cladding structure is usually formed by air holes with the same diameter arrayed in a regular triangular lattice [15-18]. The nonlinear coefficient of PCFs can be tailored by changing the air filling rate of the cladding. The chromatic dispersion profile can be easily engineered by

*Electronic address: liuzhaolun@yahoo.cn varying the holes diameter and the pitch. However, using PCFs with the entire same air-hole diameter in the cladding region, it is difficult to gain both high nonlinear coefficient and flattened dispersion coinstantaneous. We can obtain the high nonlinear coefficient PCF, and also gain flattened dispersion properties by simply adjusting the cladding parameters. But they have conditionality, when the nonlinear coefficient becomes high the dispersion is not flattened, and when the dispersion becomes flattened the nonlinear coefficient is not high. So it is necessarily to use a suited cladding structure and have a tradeoff.

We propose the PCF which has hybrid cladding with different air-holes arrayed fashions, air-holes diameters and pitches to control both the dispersion and the nonlinearity. One is the inner cladding with air-holes square array along the six axes, the other is the outer cladding with air-holes hexagon distributing as shown in Fig. 1. Where $d_{1}$ and $\Lambda_{1}$ are the air-hole diameter and the pitch of the inner cladding, $d_{2}$ and $\Lambda_{2}$ are the air-hole diameter and the pitch of the outer cladding, respectively.

The hybrid cladding can tailor the nonlinearity and dispersion of photonic crystal fibers by tuning the cladding parameters. Because of higher order diffraction from the outer cladding region, we get flattened dispersion. The inner cladding can offer facility for obtain high nonlinearity.

\section{SIMULATION RESULTS}

\subsection{Analysis method}

We used the full-vector finite element method (FEM) with the perfectly matched layer (PML) boundary conditions to analyze the nonlinearity and dispersion of the proposed hybrid cladding PCFs.

The nonlinear coefficient of PCFs and dispersion control are rested with the design of the cladding structure parameters that is condign flexibility and comparative freedom, the cladding structure parameters mostly include air-hole diameter $d$, pitch $\Lambda$ and air filling rate $f$ of cladding. The nonlinear coefficient $\gamma$ $(\lambda)$ of PCFs can be expressed as

$$
\gamma(\lambda)=2 \pi n_{2} /\left(\lambda A_{e f f}\right)
$$

where $A_{e f f}$ is model effective area, and $n_{2}=3.0 \times$ $10^{-20} \mathrm{~m}^{2} / W$, is the nonlinear index of silica. 


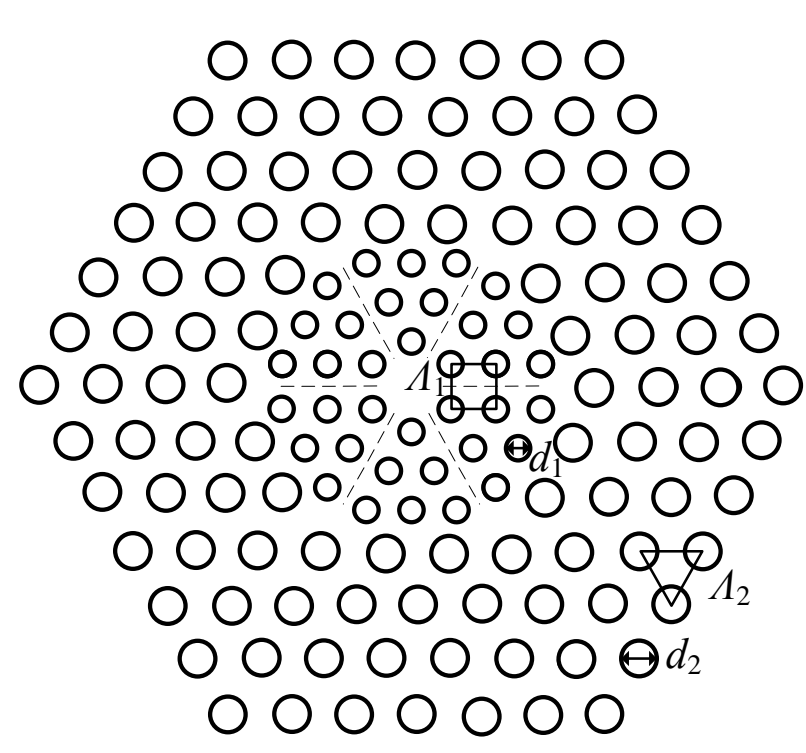

FIG. 1: The proposed hybrid cladding PCF. The inner cladding is air-holes square array along the six axes and the outer cladding is air-holes hexagon distributing.

Starting with Maxwell's curl equations, the vector equation for the magnetic field vector $\mathrm{H}$, can be derived as:

$$
\nabla \times\left(\varepsilon_{r}^{-1} \nabla \times H\right)-k_{0}^{2} \mu_{r} H=0
$$

where $\varepsilon_{r}$ and $\mu_{r}$ are the dielectric permittivity and magnetic permeability tensors, respectively. $k_{0}=2 \pi / \lambda$ is the wave number in the vacuum, $\lambda$ is operation wavelength.

The curvilinear hybrid edge/nodal elements based on linear tangential and quadratic normal vector basis functions are adopted to accomplish the computational window divisions and PML is incorporated as the boundary condition to absorb waves out of the computational window. Applying the finite element procedure to equation (2), the following eigenvalue equation

$$
[A]\{H\}=n_{\text {eff }}^{2}[B]\{H\}
$$

is obtained. Where $[A]$ and $[B]$ are the global finite element matrices. The eigenvector $\{H\}$ and the eigenvalue $n_{\text {eff }}^{2}$ provide, respectively, the full vector magnetic field distribution on the cross section of PCFs and the effective index of the mode. The dispersion of PCFs can also be divided two types, one of which is material dispersion $D_{m}(\lambda)$, and it is expressed as

$$
D_{m}(\lambda)=-\frac{\lambda}{c} \frac{d^{2} n_{m}}{d \lambda^{2}}
$$

where $n_{m}(\lambda)$ can be computed by Sellmeier formula. Other is waveguide dispersion $D_{w}(\lambda)$, and it has compact relation on the cladding structure parameters. So the total dispersion of PCFs can be expressed as

$$
D(\lambda) \approx D_{w}(\lambda)+D_{m}(\lambda)
$$

the slope of dispersion is expressed as

$$
S_{0}(\lambda)=\frac{d D}{d \lambda}
$$

The cladding regions of PCFs are considered as twodimension photonic crystal structure that possesses infinity periodicity arranged hexagon and doesn't have center defect. Toward this circular cell with symmetry boundary condition, we use the vector theory electromagnetic wave to compute. We can get the expression of waveguide dispersion $D w(\lambda)$

$$
D_{w}(\lambda)=-\frac{\lambda}{c} \frac{d^{2} \operatorname{Re}\left(n_{e f f}\right)}{d \lambda^{2}}
$$

where $n_{e f f}$ is the cladding effective index of PCFs. The variety disciplinarian of cladding effective index, $n_{e f f}$, versus $\omega$ is expressed as

$$
n_{e f f}(\omega)=\sqrt{n_{\mathrm{Si}}^{2}+u^{2}(\omega) c^{2} / \omega^{2}}
$$

\subsection{Structure analysis}

We can adjust the cladding structure parameters: $d_{1}, d_{2}, \Lambda_{1}$ and $\Lambda_{2}$ to gain high nonlinear coefficient and flattened dispersion. Above all, we analyze the relation that the nonlinear coefficient $\gamma(\lambda)$ and the waveguide dispersion $D_{w}(\lambda)$ of PCFs versus wavelength for different structure parameters.

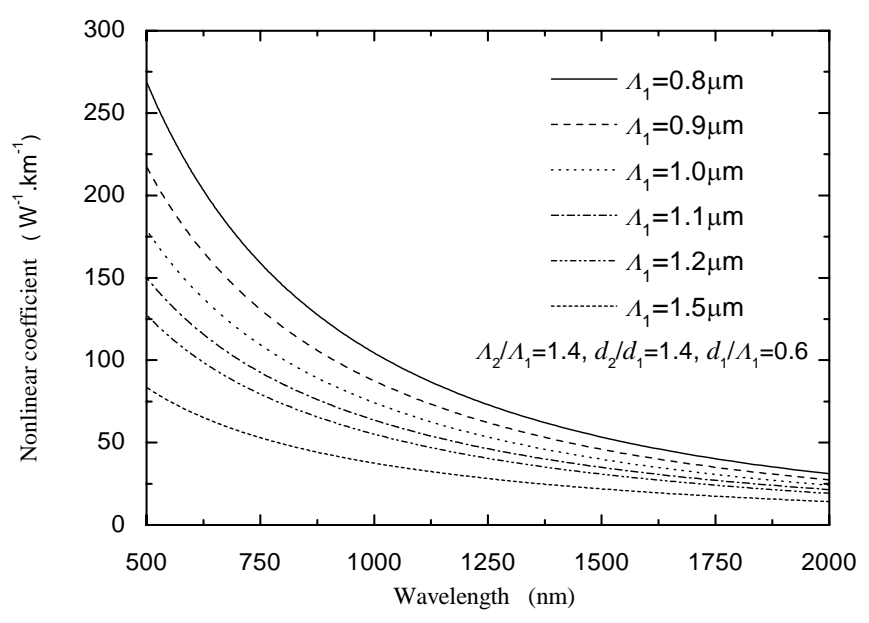

FIG. 2: Nonlinear coefficient $\gamma(\lambda)$ curves as a function of wavelength for hybrid cladding PCFs.

To keep the optimum matching of the inner cladding and the outer cladding, we set the $\Lambda_{2} / \Lambda_{1}$ is 1.4 . We firstly analyze the case when $d_{1} / \Lambda_{1}$ and $d_{2} / \Lambda_{2}$ are fixed. Fig. 2 and fig. 3 show the results for $\Lambda_{2} / \Lambda 1=1.4, d_{2} / d_{1}=1.4, d_{1} / \Lambda_{1}=$ $0.6, \Lambda_{1}$ is $0.8,0.9,1.0,1.1,1.2$ and $1.5 \mu m$ respectively. Fig. 2 shows the nonlinear coefficient versus wavelength for different $\Lambda_{1}$ and constant $d_{1} / \Lambda_{1}$. When $\Lambda_{1}$ becomes small, nonlinear coefficient increases, and it is very evidence especially at short wave. Fig. 3 is the waveguide dispersion versus wavelength, we can see when $\Lambda_{1}$ becomes small, the curve of waveguide dispersion becomes steep; the inflexion at short wave shifts up and at long wave shifts down. We can find $\Lambda_{1}$ is small, $\gamma(\lambda)$ is high, but the curve of waveguide dispersion is steep, so we select $\Lambda_{1}$ is $1.1 \mu \mathrm{m}$.

We secondly analyze the case when $\Lambda_{1}$ is fixed for different $d_{1} / \Lambda_{1}$, and we also set the $\Lambda_{2} / \Lambda_{1}$ is 1.4. Fig. 4 and fig. 5 


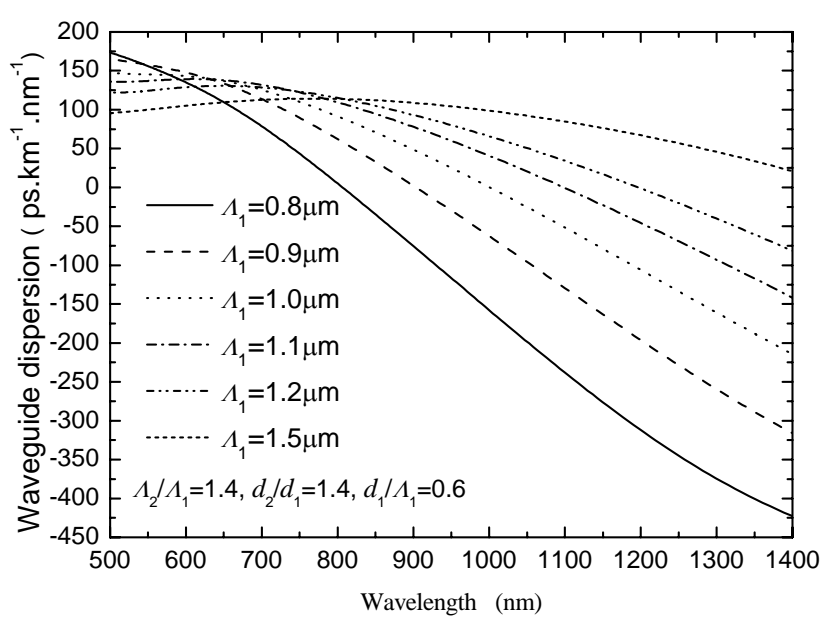

FIG. 3: Waveguide dispersion $D_{w}(\lambda)$ curves as a function of wavelength for hybrid cladding PCFs.

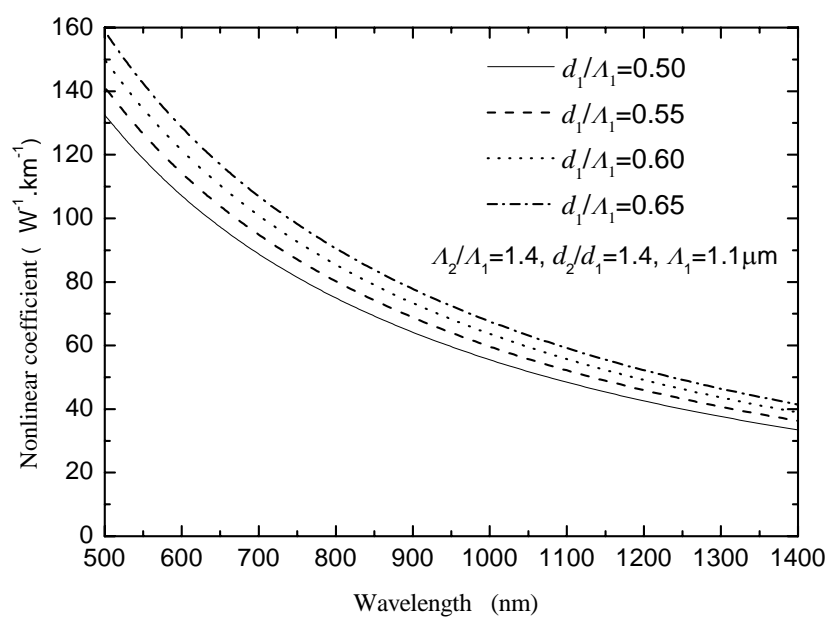

FIG. 4: Nonlinear coefficient curves as a function of wavelength for hybrid cladding PCFs.

show the simulating result $d_{1} / \Lambda_{1}$ is $0.5,0.55,0.6$, and 0.65 respectively. Where fig. 4 is the nonlinear coefficient versus wavelength, and fig. 5 is the waveguide dispersion versus wavelength. From fig. 4 , we can see when the $d_{1} / \Lambda_{1}$ increases, the nonlinear coefficient also increases. From fig. 5, we can see when $d_{1} / \Lambda_{1}$ increases, the waveguide dispersion curve shifts along long wave and shifts up, but it has little influence on the tendency of the waveguide dispersion curves. We can find $d_{1} / \Lambda_{1}$ is large, $\gamma(\lambda)$ is high, but the curve of waveguide dispersion shifts along long wave and different to keep the total dispersion flattened, so we select $d_{1} / \Lambda_{1}$ is 0.6 .

We lastly analyze the case when $\Lambda_{1}$ and $d_{1} / \Lambda_{1}$ are fixed, and $d_{2} / \Lambda_{2}$ is not equal to $d_{1} / \Lambda_{1}$. Fig. 6 and fig. 7 show the simulating result for the case that $\Lambda_{1}$ is $1.1 \mu \mathrm{m}, \Lambda_{2} / \Lambda_{1}=1.4$, $d_{1} / \Lambda_{1}=0.6, d_{2} / \Lambda_{2}=0.60,0.65,0.70$, and 0.75 , respectively. Where fig. 6 is the nonlinear coefficient versus wavelength, and fig. 7 is the waveguide dispersion versus wave-

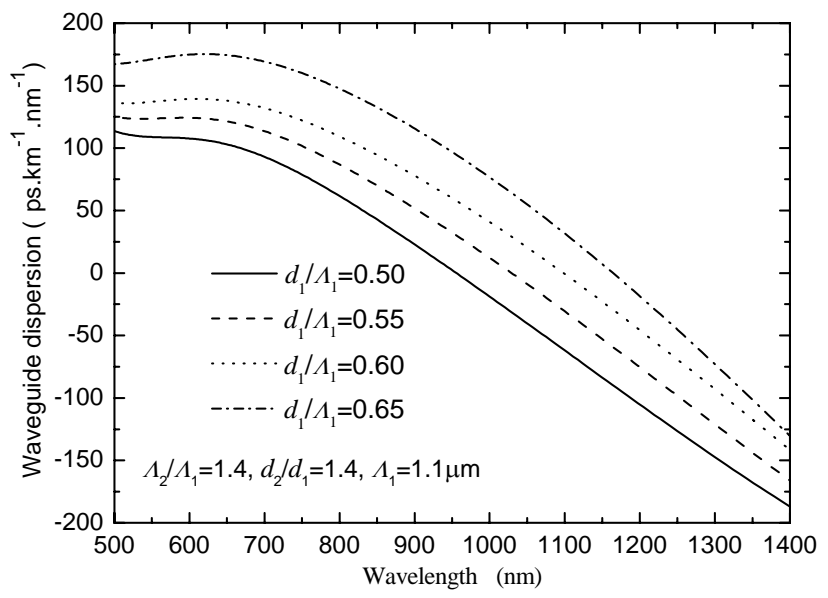

FIG. 5: Waveguide dispersion curves as a function of wavelength for hybrid cladding PCFs.

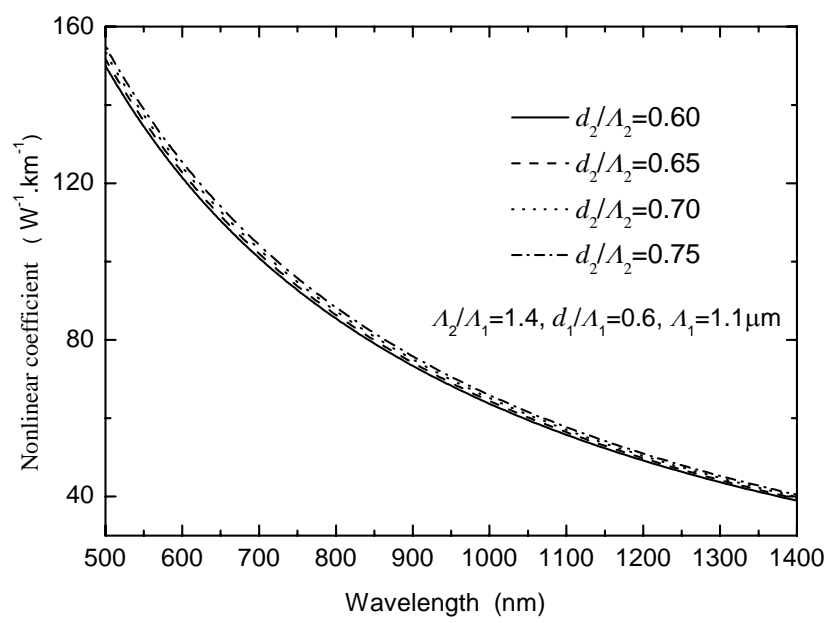

FIG. 6: Nonlinear coefficient curves as a function of wavelength for hybrid cladding PCFs.

length. From fig. 6, we can see when the $d_{2} / \Lambda_{2}$ increases, the nonlinear coefficient also increases, but the increment is less. From fig. 7, we can see when $d_{2} / \Lambda_{2}$ increases, the waveguide dispersion curve only has a little change. We can find $d_{2} / \Lambda_{2}$ is large, $\gamma(\lambda)$ is high, and the curve of waveguide dispersion is also suitable for holding the total dispersion flattened, so we select $d_{2} / \Lambda_{2}$ is 0.75 .

Based on the analysis above, we can find that the cladding structure parameters $\Lambda_{1}, d_{1} / \Lambda_{1}$ and $d_{2} / \Lambda_{2}$ all have influence on the nonlinear coefficient and the waveguide dispersion. The pitch $\Lambda_{1}$ has decisive influence on the value of nonlinear coefficient and the tendency of waveguide dispersion curves, $d_{1} / \Lambda_{1}$ also influences the value of nonlinear coefficient and the size and the position of waveguide dispersion, and $d_{2} / \Lambda_{2}$ generally influences the value of nonlinear coefficient and has a little effect on the size and position of waveguide dispersion. By reducing $\Lambda_{1}$ or increasing $d_{1} / \Lambda_{1}$, we can obtain high nonlinear coefficient of PCFs, and properly increasing $d_{2} / \Lambda_{2}$ can obtain 
higher nonlinear coefficient and maintain the dispersion flattened.

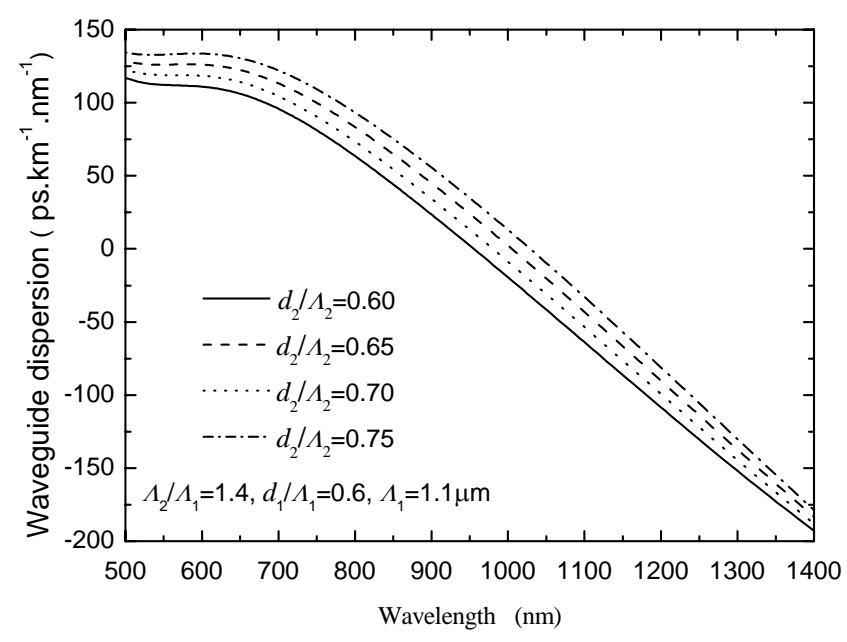

FIG. 7: Waveguide dispersion curves as a function of wavelength for hybrid cladding PCFs.

\subsection{Adjusted results}

Now we have an example that designs PCFs with high nonlinearity and flattened dispersion properties. In order to predigest designing, the total dispersion are expressed as

$$
D(\lambda) \approx D_{w}(\lambda)-\left(-D_{m}(\lambda)\right)
$$

where $D_{m}(\lambda)$ is the material dispersion. Because the diversification of material dispersion versus wavelength don't suffer the influence of the cladding structure parameters, so we can obtain flattened dispersion properties by adjusting $D_{w}(\lambda)$ to balance $-D_{m}(\lambda)$. Combining the computed result above, we can separate the design of flattened dispersion high nonlinearity PCF into three steps. Firstly, by adjusting $\Lambda_{1}$ we can find the tendency of waveguide dispersion that close the diversification of $-D_{m}(\lambda)$ curves, secondly, by changing $d_{1} / \Lambda_{1}$ we can adjust waveguide dispersion, thirdly properly increasing $d_{2} / \Lambda_{2}$ we can gain higher nonlinear coefficient, finally we can properly change $\Lambda_{1}, d_{1} / \Lambda_{1}$ and $d_{2} / \Lambda_{2}$ to obtain flattened dispersion high nonlinearity PCF.

Fig. 8 is the total chromatic dispersion, wave dispersion and material dispersion for modified structure parameters that we design for gaining high nonlinearity dispersion flattened hybrid cladding PCF. This high nonlinearity dispersion flattened hybrid cladding PCF has the cladding structure parameters: $\Lambda_{1}=1.1 m, \Lambda_{2} / \Lambda 1=1.4, d_{1} / \Lambda_{1}=0.6$, and $d_{2} / \Lambda_{2}=0.75$. From fig. 8 , we can see this hybrid cladding PCF has a flattened dispersion profile.

By adjusting the cladding structure parameters, we can obtain the high nonlinearity dispersion flattened hybrid cladding PCF. Fig. 9 shows three high nonlinearity dispersion flattened hybrid cladding PCF with different structure parameters. The high nonlinearity hybrid cladding PCF A has the structure parameters: $\Lambda_{1}=1.1 \mu m, d_{1}=0.65 \mu m, \Lambda_{2}=1.54 \mu m$, and $d_{2}=$

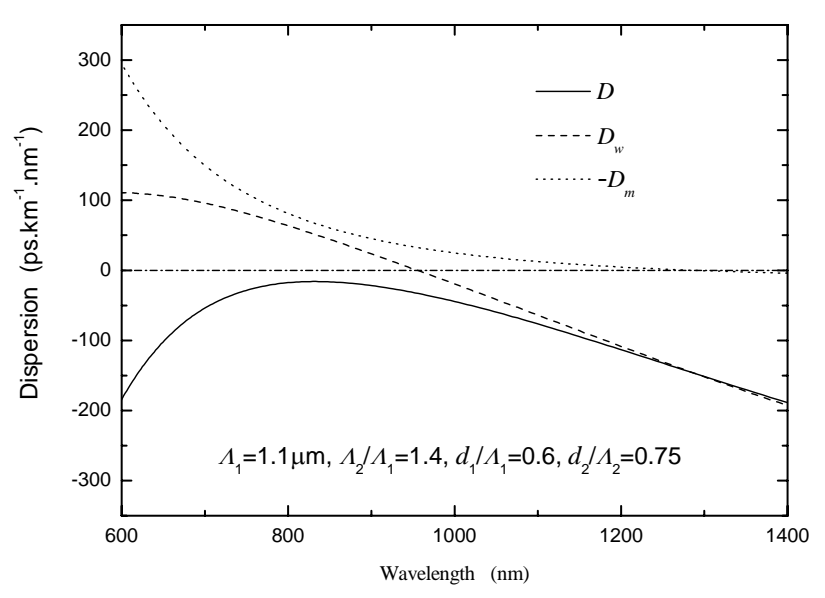

FIG. 8: The total chromatic dispersion, wave dispersion and material dispersion curves as a function of wavelength for hybrid cladding PCF.

$1.05 \mu \mathrm{m}$; the high nonlinearity hybrid cladding PCF B has the structure parameters: $\Lambda_{1}=1.1 \mu, d_{1}=0.65 \mu \mathrm{m}, \Lambda_{2}=1.55 \mu \mathrm{m}$, and $d_{2}=1.15 \mu \mathrm{m}$; the high nonlinearity hybrid cladding $\mathrm{PCF}$ $\mathrm{C}$ has the structure parameters: $\Lambda_{1}=1.1 \mu \mathrm{m}, d_{1}=0.65 \mu \mathrm{m}$, $\Lambda_{2}=1.58 \mu \mathrm{m}$, and $d_{2}=1.25 \mu \mathrm{m}$. The hybrid cladding PCFs all have high nonlinearity and flattened dispersion, and PCF $\mathrm{A}, \mathrm{PCF} \mathrm{B}$ and PCF $\mathrm{C}$ has negative dispersion, near zero dispersion and positive dispersion around $800 \mathrm{~nm}$, respectively.

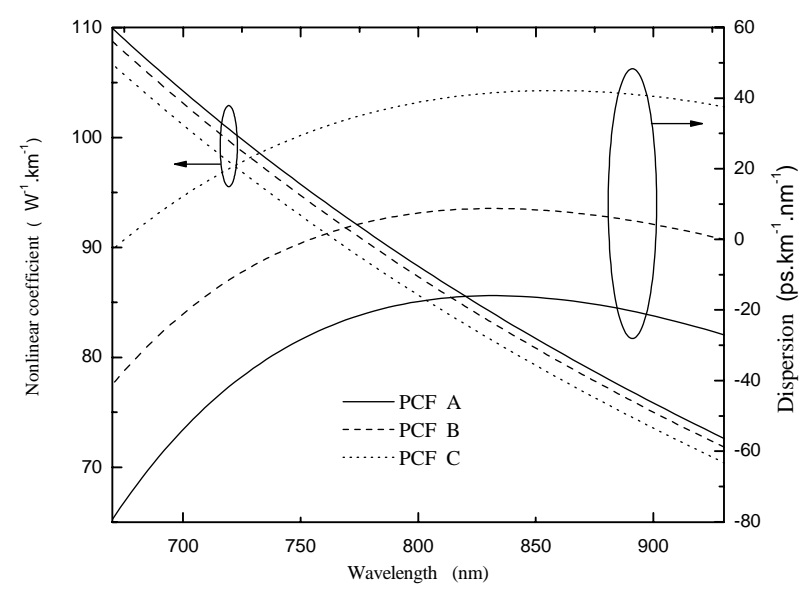

FIG. 9: Nonlinear coefficient curves and the total dispersion curves as a function of wavelength for hybrid cladding PCFs.

We can obtain high nonlinearity PCFs by adjusting the structure parameters of the proposed hybrid cladding PCF. The hybrid cladding PCFs have high nonlinearity and flattened dispersion from $630 \mathrm{~nm}$ to $970 \mathrm{~nm}$. The hybrid cladding PCF can have flattened dispersion, which can bring spectral broadening through fission of higher-order solitons [19-21]. The hybrid cladding PCF have the important action for by using Ti: Sapphire laser to study the nonlinear effect of ultra short laser pulses in PCF. So we proposed hybrid cladding PCF with high 
nonlinear coefficient and dispersion flattened which is suited for supercontinuum generation.

\section{CONCLUSIONS}

Using a vectorial FEM, we have computed the nonlinear coefficient and the dispersion properties of a novel PCF with hybrid cladding which has different pitches and air-holes diameters. The results indicate that $\Lambda_{1}$ have decisive influence on the tendency of waveguide dispersion curves, and $d_{1} / \Lambda_{1}$ generally influences the size and position of waveguide dispersion. By reducing $\Lambda_{1}$ or increasing $d_{1} / \Lambda_{1}$, we can obtain high nonlinear coefficient of PCFs, and properly increasing $d_{2} / \Lambda_{2}$ can obtain higher nonlinear coefficient and maintain the dispersion flattened. Using a hybrid cladding structure and adjusting the cladding structure parameters, we have obtained the high nonlinearity dispersion flattened PCF. The hybrid cladding PCF we proposed is capable to possess of both high nonlinear coefficient and flattened dispersion. It is useful for the hybrid cladding PCF for their applications in nonlinear fiber optics.

\section{Acknowledgements}

The work is supported by the National Natural Science of China with granted No. 60637010.
[1] J. Knight, Nature 424, 847 (2003).

[2] P. Russell, Science 299, 358 (2003)

[3] K. Chow, Y. Takushima, and C. Lin, Electronics Letters, 42, 989 (2006).

[4] Z. Zhu and T. Brown, J. Opt. Soc. Am. B, 21, 249 (2004).

[5] K. Abedin and F. Kubota, Electron. Lett 40, 5 (2004).

[6] S. K. Varshney, T. Fujisawa, K. Saitoh, and M. Koshiba, Optics Express, 13, 9516 (2005).

[7] A. Zheltikov, Applied Physics B: Lasers and Optics, 84, 69 (2006).

[8] J.Y.Y. Leong, P. Petropoulos, J.H.V. Price, Heike EbendorffHeidepriem, S. Asimakis, R.C. Moore, K. E. Frampton, V. Finazzi, X. Feng, T. M. Monro, and D.J. Richardson, J. Lightwave Technol. 24, 183 (2006).

[9] K. Saitoh, N. Florous and M. Koshiba, Opt. Lett., 31, 26 (2006).

[10] K.K. Chow, C. Shu, Senior Member, Chinlon Lin, and A. Bjarklev, Photon. Technol. Lett. 17, 624 (2005).

[11] J.M. Dudley and S. Coen, Opt. Express, 12, 2423 (2004).

[12] N. Nishizawa, Y. Ito, and T. Goto, Jpn. J. Appl. Phys. 42, 449 (2003).
[13] S. Haxha, and H. Ademgil, Optics Communications 281, 278 (2008).

[14] J. Wang, C. Jiang, W. Hua, and M. Gao, Optics \& Laser Technol. 38, 169 (2006).

[15] S.M. Abdur Razzak, and Yoshinori Namihira, Photon. Technol. Lett. 20, 249 (2008).

[16] M. Chen, S. Xie, Optics Communications 281, 2073 (2008).

[17] N. Florous, K. Saitoh, and M. Koshiba, Opt. Express, 14, 901 (2006).

[18] F. Poli, A. Cucinotta, and S. Selleri, Photon. Technol. Lett. 16, 1065 (2004).

[19] J. M. Dudley, G. Genty, and S. Coen, Rev. Mod. Phys. 78, 1135 (2006).

[20] J. Herrmann, U. Griebner, N. Zhavoronkov, A. V. Husakou, D. Nickel, J. C. Knight, W. J. Wadsworth, P. St. J. Russell, and G. Korn, Phys. Rev. Lett. 88, 173901 (2002).

[21] A.V. Husakou, and J. Herrmann, Phys. Rev. Lett. 87, 203901 (2001). 